Article

\title{
Study on the Coupling Mechanism of the Orthogonal Dipoles with Surface Plasmon in Green LED by Cathodoluminescence
}

\author{
Yulong Feng ${ }^{1}$, Zhizhong Chen ${ }^{1,2, *}$, Shuang Jiang ${ }^{1}$, Chengcheng Li ${ }^{1}$, Yifan Chen ${ }^{1}$,

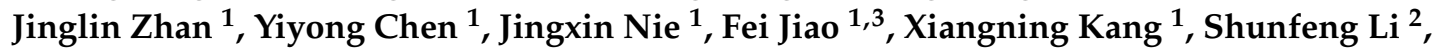 \\ Tongjun $\mathrm{Yu}^{1}{ }^{1}$, Guoyi Zhang ${ }^{1,2}$ and Bo Shen ${ }^{1}$ \\ 1 State Key Laboratory for Artificial Microstructure and Mesoscopic Physics, School of Physics, \\ Peking University, Beijing 100871, China; fengyulong@pku.edu.cn (Y.F.); jiangshuang@pku.edu.cn (S.J.); \\ 1501110124@pku.edu.cn (C.L.); 1501110129@pku.edu.cn (Y.C.); 1601110180@pku.edu.cn (J.Z.); \\ chenyiyong@pku.edu.cn (Y.C.); niejingxin@pku.edu.cn (J.N.); fjiao@pku.edu.cn (F.J.); \\ xnkang@pku.edu.cn (X.K.); tongjun@pku.edu.cn (T.Y.); gyzhang@pku.edu.cn (G.Z.); \\ bshen@pku.edu.cn (B.S.) \\ 2 Dongguan Institute of Optoelectronics, Peking University, Guangdong, Dongguan 523808, China; \\ shunfengli@gmail.com \\ 3 State Key Laboratory of Nuclear Physics and Technology, School of Physics, Peking University, \\ Beijing 100871, China \\ * Correspondence: zzchen@pku.edu.cn; Tel.: +86-10-6275-2169
}

Received: 23 March 2018; Accepted: 12 April 2018; Published: 16 April 2018

\begin{abstract}
We analyzed the coupling behavior between the localized surface plasmon (LSP) and quantum wells (QWs) using cathodoluminescence (CL) in a green light-emitting diodes (LED) with Ag nanoparticles (NPs) filled in photonic crystal (PhC) holes. Photoluminescence (PL) suppression and CL enhancement were obtained for the same green LED sample with the Ag NP array. Time-resolved PL (TRPL) results indicate strong coupling between the LSP and the QWs. Three-dimensional (3D) finite difference time domain (FDTD) simulation was performed using a three-body model consisting of two orthogonal dipoles and a single Ag NP. The LSP-QWs coupling effect was separated from the electron-beam (e-beam)-LSP-QW system by linear approximation. The energy dissipation was significantly reduced by the z-dipole introduction under the e-beam excitation. In this paper, the coupling mechanism is discussed and a novel emission structure is proposed.
\end{abstract}

Keywords: localized surface plasmon; green LED; cathodoluminescence; FDTD

\section{Introduction}

Despite GaN-based blue light-emitting diodes (LEDs) achieving rather high external quantum efficiency (EQE), the green gap is still a key issue for high-quality illumination [1,2]. Because of the imperfect structure and high polarization field in high-indium content InGaN quantum wells (QWs), the EQE for a green LED is quite low compared with that for a blue LED. Various approaches have been applied to solve this problem, such as the use of nonpolar/semipolar substrates, the use of a Si substrate, band engineering, and the introduction of a surface plasmon (SP) [3-6]. The polarization reduction and incorporation of indium into the InGaN alloy have been improved by these methods except for the last one, the SP method. Because of the high density of states (DOS) of the SP, the spontaneous emission rate (SER) in QWs can be very high, leading to a higher internal quantum efficiency (IQE) [6]. Two main perspectives on light emission enhancement via SP-QW coupling have emerged. One perspective is that the SP modes in the metal are excited by spontaneous emission (SE) in QWs and radiate to 
air in dipole mode [7,8], thus the energy transferred to SP is divided into "SP radiation" and "metal dissipation". The other perspective is that the spontaneous emission rate in QWs is greatly enhanced by the strong near-field strength of the SP excited by SE in QWs [9-11]. Both of these perspectives can explain the light emission enhancement by SP-QW coupling. As to SP radiation, less than $50 \%$ of the coupled energy can be radiated into air, and the emission enhancement is effective for emitters with low original EQE [8]. The latter perspective introduces the possibility of emission enhancement for a green LED $[10,11]$. However, the energy distribution dynamics in the SP-QW coupling system remain unclear.

Although evidence of the SER enhancement has been reported for several decades, the problem of energy dissipation in the metal is not yet well resolved. The energy dissipation in the metal corresponds to the Ohmic loss and to the electron-hole pair creation [12]. Many techniques have been reported to reduce the energy dissipation effects, including using a localized surface plasmon (LSP), placing the emitters into a metal gap, adjusting the dipole orientation, and coupling the QWs between each other $[8,10,11,13,14]$. An enhancement of the SER exceeding 1000 times with a quantum efficiency above $50 \%$ indicates that the energy dissipation is greatly reduced by the gap modes [10]. However, the complicated fabrication procedure for the gap structure prevents its further application. LSP-QW coupling between the radial or orbital dipoles and Ag NPs is quite different in the simulation [13]. The enhancements of radiated power of a radial and an orbital dipole are induced through coupling with the lower-order (dipole) and higher-order LSP resonance, respectively. Because the conventional InGaN QWs are thin and flat, the dipoles are mainly orbitally oriented $[9,13]$, that is, some energy dissipation always occurs for planar QW structure.

Recently, several reports of electron beam (e-beam)-excited SP in metal nanoparticles (NPs) by cathodoluminescence (CL) have been reported [15-18]. Because CL measurements combine the ultrahigh spatial resolution of an electron microscope with broadband optical sensitivity, they can be used to study the optical process in metal NPs. There is a direct link between CL and radiative modes ("bright modes") or the radiative electromagnetic local density of states (LDOS) [16]. Some simulation results for CL measurements have also been performed by regarding the e-beam as a dipole source along the incident direction [15,18]. Although Ag NP-QW structures excited by an e-beam have been reported [19-21], these works did not consider the SPs induced by the e-beam. In fact, the e-beam, approximated by a vertical dipole induces the high near-field strength near the Ag NPs [15-18], will greatly influence the coupling between the LSP and the QWs. Moreover, the dipoles representing the QW and the e-beam are in-plane and out-plane, respectively. The configuration of a single Ag $\mathrm{NP}$ and two orthogonal dipoles can be used to study the energy transfer process of the LSP-QW coupled system.

In this work, we fabricated LSP-QW coupled samples of an array of Ag NPs embedded in photonic crystal (PhC) holes in the p-GaN layer of a green LED. Photoluminescence (PL) and time-resolved photoluminescence (TRPL) and CL measurements were carried out. A novel three-dimensional (3D) finite difference time domain (FDTD) numerical simulation model for CL and PL measurements was also put forward using Lumerical software (FDTD Solutions v8.17, Vancouver, BC, Canada) to illustrate the difference in the LSP-QW coupling mechanism under light and e-beam excitations [22]. The LSP-two orthogonal dipoles coupling mechanism is discussed and an effective way to reduce the energy dissipation in Ag NPs is proposed.

\section{Experimental}

GaN-based green LED structures with a peak wavelength of $545 \mathrm{~nm}$ were grown by metal organic chemical vapor deposition (MOCVD) on the double-polished c-plane sapphire substrate. The structure consisted of 10 pairs of InGaN/GaN (2.5 nm/12.5 nm) multiple quantum wells (MQWs), on which is $160 \mathrm{~nm}$ thick p-GaN. As shown in Figure 1, LSP-QW coupled samples (Ag-PhC) were fabricated based on the PhC structure in the $\mathrm{p}-\mathrm{GaN}$ layer. The scanning electron microscope (SEM) images of the Ag arrayed LED were recorded using an FEI NanoSEM 430 (FEI, Hillsboro, OR, USA). First, 
a $120 \mathrm{~nm}$ thick $\mathrm{SiO}_{2}$ mask was deposited onto the surface of $\mathrm{p}-\mathrm{GaN}$. The PhC patterns were obtained via nano-imprinting using an Obducat Eitre 3 instrument (Obducat, Lund, Sweden) with a standard $\mathrm{PhC}$ stamp whose period was $545 \mathrm{~nm}$. Then, the hexagonal nanohole array was subsequently obtained using an induced coupled plasma (ICP) etcher to etch the pores to a depth of $150 \mathrm{~nm}$, i.e., the bottom of each pore was $10 \mathrm{~nm}$ away from QWs. Then, a $30 \mathrm{~nm}$ thick Ag film was deposited onto the patterned surface. After thermal annealing at $600{ }^{\circ} \mathrm{C}$ for $10 \mathrm{~min}$ under a $\mathrm{N}_{2}$ atmosphere, Ag NPs were formed in the PhC holes, followed by the lift-off process to remove the $\mathrm{Ag} \mathrm{NPs}$ on the $\mathrm{SiO}_{2}$ mask. The $\mathrm{Ag}$ NPs were spherical cap-shaped. Statistically, the diameter and height were $160 \pm 10$ and $\sim 80 \mathrm{~nm}$, respectively. For comparison, reference samples (denoted $\mathrm{PhC}$ ) with the same pattern but without $\mathrm{Ag}$ NPs were also prepared.

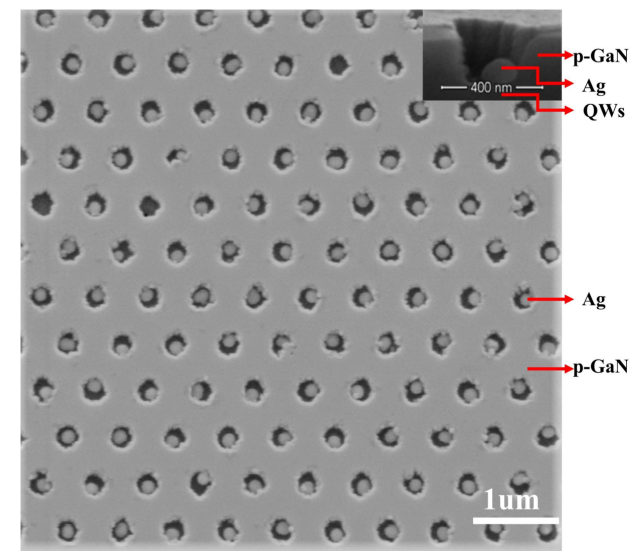

Figure 1. SEM image of a Ag-photonic crystal $(\mathrm{PhC})$ sample. The period of the $\mathrm{PhC}$ is $545 \mathrm{~nm}$. The diameter and height of the Ag NPs are $160 \pm 10$ and $\sim 80 \mathrm{~nm}$, respectively. The inset shows the cross-sectional image of a single Ag NP in the hole.

PL measurements using a $405 \mathrm{~nm}$ laser diode with a power of $150 \mathrm{~mW}$ and a spot diameter of $\sim 1 \mathrm{~mm}$ and CL measurements using a Gatan Mono-CL2 system (Gatan, Pleasanton, CA, USA) were performed at room temperature on the $\mathrm{Ag}-\mathrm{PhC}$ and $\mathrm{PhC}$ samples with a configuration of top excitation and top detection. The electron acceleration voltage was set to $15 \mathrm{kV}$ with a beam current of $158 \mathrm{pA}$. The TRPL measurements were conducted using a LifeSpec-Red picosecond lifetime spectrometer with a pulsed $372 \mathrm{~nm}$ laser (Edinburgh Instruments, Livingston, UK). The pulse duration was 69 ps. This instrument was made use of a time-correlated single photon counting (TCSPC) technique with a time resolution of $\sim 30$ ps within a range of $10 \mathrm{~ns}$.

\section{Results and Discussion}

TRPL measurement is an efficient method to confirm LSP-QW coupling. Figure 2A shows the TRPL results at the peak wavelength $545 \mathrm{~nm}$ of the $\mathrm{Ag}-\mathrm{PhC}$ sample and the PhC sample. The decay curves were fitted by a double exponential function as reported in the previous work [14]. The fast decay time, which corresponds to the rapid carrier recombination in InGaN QWs, were obtained as 0.23 and $0.76 \mathrm{~ns}$ for the $\mathrm{Ag}-\mathrm{PhC}$ and $\mathrm{PhC}$ samples, respectively. The 3.3-fold reduction of the decay time indicates that LSP coupling with QWs substantially enhances the SER [14]. Contrarily, the PL intensity of the $\mathrm{Ag}-\mathrm{PhC}$ decreased by 1.7 times compared with that of PhC sample, as shown in Figure 1B. This decrease is attributed to the large energy dissipation in Ag NPs as a result of their small aspect parameter $(\alpha)$, which is defined as the ratio between the height of the Ag NP and its radius [14,23]. When the $\alpha$ is greater than 1.5, the emission enhancement will be realized with $\mathrm{Ag}$ NPs with diameters ranging from 90 to $200 \mathrm{~nm}$ [23]. Because the LSP-QW coupled energy was either radiated into the air or dissipated in the Ag NPs, the ratio between radiated energy and the dissipated energy would determine whether the final PL intensity was enhanced or suppressed. 
A

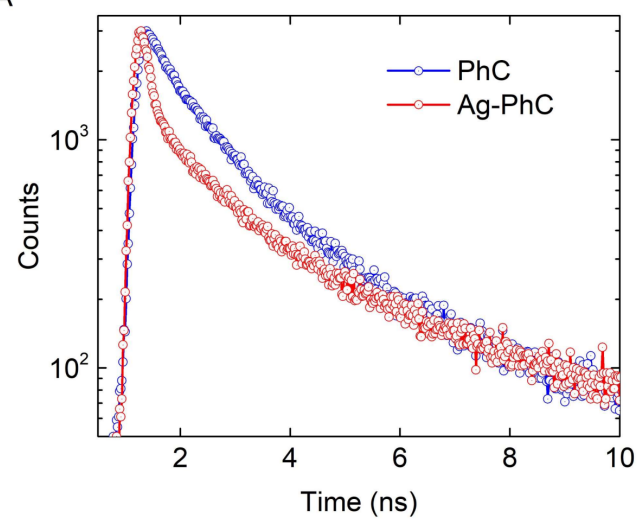

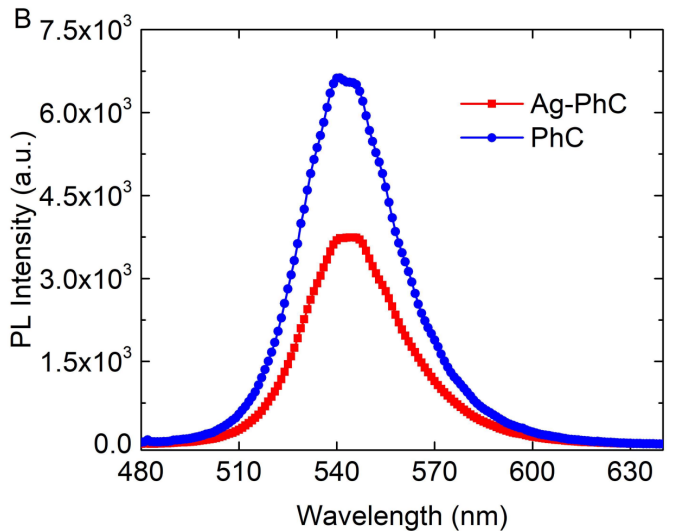

Figure 2. (A) Time-resolved photoluminescence (TRPL) and (B) PL spectra for Ag-PhC and PhC samples.

A schematic setup for the CL measurement is shown in Figure 3A. An e-beam was highly focused and directed onto the surface of samples. CL measurement was performed using a $15 \mathrm{kV}$ acceleration voltage and a beam current of $158 \mathrm{pA}$. The electron penetrating depth into the Ag NPs was greater than $20 \mathrm{~nm}[19,20]$. The light emitted from the samples was collected by a retractable parabolic mirror and collimated to an optical monochromator, after which the signal was detected by a charge-coupled device (CCD). To ensure the maximum light collection efficiency, the samples were placed at the focal plane of the parabolic mirror, approximately $\sim 1 \mathrm{~mm}$ away from the mirror. Figure 3C,D show the panchromatic CL (PanCL) image of an Ag-PhC sample and a PhC sample, respectively. The PanCL image, where all of the emitted light from the sample was collected by the e-beam scanning point by point, can clearly depict the LSP-induced luminescence around the Ag NPs. The holes are much brighter than the platform of the Ag-PhC sample. The dark spots in the holes are related to the Ag NPs. However, the spot size is much smaller than the actual size of Ag NPs in Figure 1. This discrepancy arises because of the penetration ability of high-energy electrons [19] and the strong coupling between the LSPs induced by the e-beam [16-18] and the QWs. The darker cloudy areas in Figure 3C,D are mainly attributed to the InGaN phase separation in QWs. CL spectra were recorded by scanning the electron beam over the entire surface of interest under the same conditions, as shown in Figure 3B. To confirm that the emission originates from QWs rather than from the Ag NPs, samples without QWs were fabricated. It was found that CL spectrum of the sample without QWs mainly originated from the emission of $\mathrm{GaN}$ and its intensity was approximately two orders of magnitude smaller than that of the sample with QWs. The CL intensity of the Ag-PhC sample is 2.91 times greater than that of the PhC sample, whereas the PL intensity of PhC sample is 1.7 times that of the $\mathrm{Ag}-\mathrm{PhC}$ sample. Compared with laser excitation, e-beam excitation enhances the emission intensity of the $\mathrm{Ag}-\mathrm{PhC}$ sample by a factor of 4.95. Considering the penetration electron energy loss in Ag NPs, even if all the electrons can penetrate through the Ag NP to excite the QW directly, it is impossible for the enhancement factor to be as high as 4.95. The high near-field strength of the LSP in the Ag NPs induced by continuously injected e-beam should be considered in the LSP-QW coupling process [16-18]. Moreover, how the energy dissipation is reduced by the e-beam excitation is interesting. 


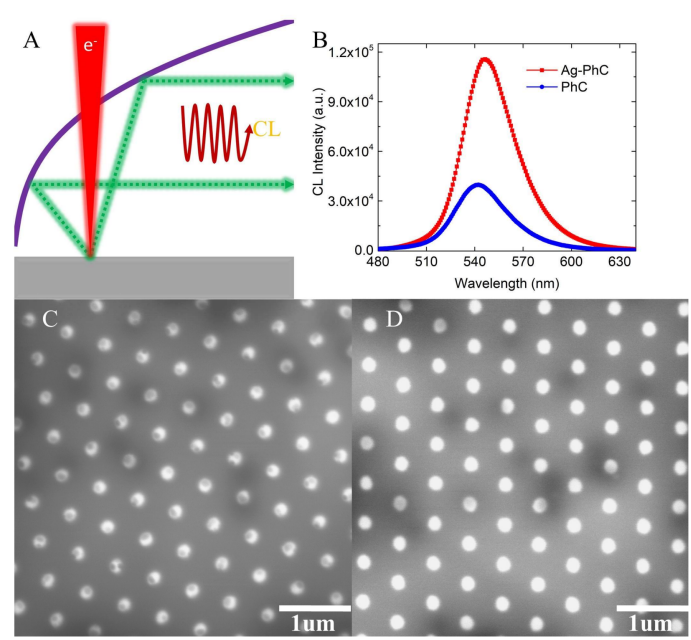

Figure 3. (A) Schematic setup for the cathodoluminescence (CL) measurement; (B) CL spectra for $\mathrm{Ag}-\mathrm{PhC}$ and $\mathrm{PhC}$ samples. Panchromatic CL images for (C) the Ag-PhC sample and (D) the PhC sample. CL intensity for the $\mathrm{Ag}-\mathrm{PhC}$ sample is enhanced 2.91 times compared with that for PhC sample.

To distinguish the different LSP-QW coupling mechanisms by e-beam excitation and laser excitation, 3D-FDTD numerical simulations were carried out [22]. In FDTD, Maxwell's equations are solved in discretized space and time. Figure 4 shows the schematic structure of Ag-PhC sample used in the 3D-FDTD simulation. Since the separation between Ag NPs is greater than $200 \mathrm{~nm}$, the coupling between Ag NPs can be ignored [24]. Therefore, only one Ag NP needs to be considered. The dispersion relation of the Ag NP adopts "Ag (silver)-Palik (0-2 um)" data provided by the FDTD material database [22]. The Ag NP was placed in the hole center of PhC on p-GaN $(n=2.55)$ to simplify the simulation. The sizes of Ag NP and PhC are consistent with the SEM result as shown in Figure 1. The space between Ag NP and the first QW is $10 \mathrm{~nm}$. The perfectly matched layer (PML) absorbing boundaries were adopted on all sides. To improve the simulation accuracy, an override region was applied over the Ag NPs and x-dipole with the mesh size of $2 \mathrm{~nm}$. Outside that region, automatic graded meshing was used. Moreover, the simulation span was 6 um $^{*} 6$ um (x-y plane), which is large enough for the light to propagate.

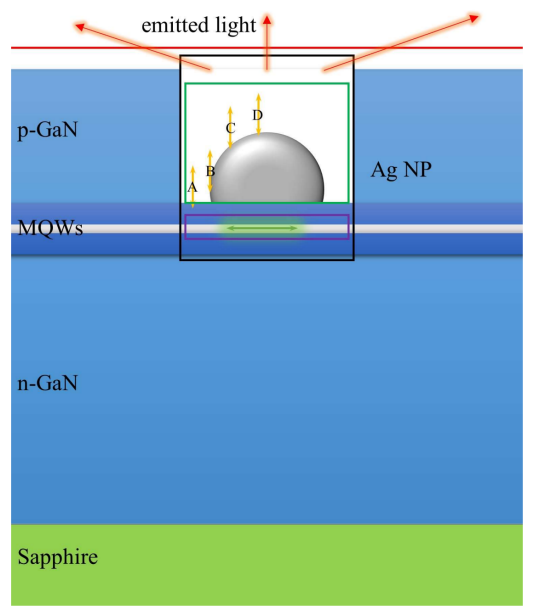

Figure 4. The schematic structure of the Ag-PhC sample in 3D finite difference time domain (FDTD) simulation. The purple, green, and black boxes were used to collect the total power radiated by the dipole, the dissipation power in the Ag NP, and the scatted energy, respectively. The red line (plane) was used to record the radiated power from top surface. 
Given the symmetry in the QW plane, where the radiating dipoles lie, only one dipole polarized along $x$ direction (x-dipole) was placed below the Ag NP to represent the QW. The e-beam, however, was represented by a dipole polarized along its trajectory (z-dipole) in order to simplify the model [18]. Both the $x$-dipole and z-dipole adopted the built-in broadband source model, with the wavelength range from 480 to $630 \mathrm{~nm}$. In FDTD, broadband sources can be used to perform simulations in which wideband frequency data is required. Since the e-beam impinging on different positions of the Ag NP may lead to different excitation, the z-dipole was successively placed with an interval of $30 \mathrm{~nm}$ at the positions of A, B, C, and D, as shown by the yellow arrows in Figure 4. To calculate the power transition, four monitors were used in the simulations. The purple, green, and black boxes were used to collect the power of $x$-dipole $(\mathrm{QW})$, dissipated power, and scattering power, respectively, while the upper red plane monitor was used to record the energy successfully extracted into the air.

The actual power emitted by a dipole can vary dramatically depending on what dielectric envelopments are nearby. The field induced by a dipole or reflected from the surrounding structures can feed back on itself, causing it to radiate more or less power than expected in a homogenous material $[10,11,13]$. As a result, the calculated power should be normalized to the power that a dipole would emit in a homogenous material (such as vacuum), rather than the actually emitted power. In FDTD, the quantum mechanical decay rate can be related to the power collected by a monitor box in the same environment. Therefore, the decay rate can be normalized by [22],

$$
\frac{\gamma}{\gamma_{0}}=\frac{P}{P_{0}}
$$

where $\gamma$ and $P$ are the decay rate and radiated power by a dipole in an inhomogeneous environment, and $\gamma_{0}$ and $P_{0}$ are the corresponding parameters in a homogeneous environment (here GaN). In particular, when a dipole is surrounded by a monitor box (i.e., the purple box in Figure 4 ), $\gamma$ would become the well-known Purcell factor $\left(F_{p}\right)$, which is defined as the ratio of the radiation power of a dipole near Ag NPs to that in a bulk dielectric material (GaN). Thus, the EQE-namely the ratio of power measured in the far field to the total power injected into the $x$-dipole (QW) - as well as the IQE and light extraction efficiency (LEE) can be defined as [11,22],

$$
\eta_{E Q E}=\eta_{I Q E} \eta_{L E E}=\left(\frac{F_{p} \gamma_{\text {rad }}}{F_{p} \gamma_{\text {rad }}+\gamma_{\text {non }}} \frac{\gamma_{\text {scat }}}{\gamma_{\text {diss }}+\gamma_{\text {scat }}}\right) \frac{\gamma_{\text {out }}}{\gamma_{\text {scat }}}
$$

where $\gamma_{\text {scat }}$ and $\gamma_{\text {diss }}$ are the scattering and dissipation rate, $\gamma_{\text {rad }}$ and $\gamma_{\text {non }}$ are the radiative and nonradiative decay rate, and $\gamma_{o u t}$ is light extraction rate. According to our temperature-dependent PL measurements, the internal quantum efficiency is calculated as $26 \%$. A rough estimation of the ratio of the nonradiative decay rate $\left(\gamma_{n o n}\right)$ to the radiative decay rate $\left(\gamma_{r a d}\right)$ for the $\mathrm{PhC}$ sample is 3:1. Based on the data drawn from the aforementioned monitors, EQE, IQE, LEE, and $F_{p}$ can be calculated, respectively.

In the PL simulation, because the Ag NPs are opaque to light excitation [19] or the laser wavelength cannot match the SP resonant wavelength [14], z-dipole is not included, similar to our previous work [11,23]. Figure 5 shows the simulated PL spectra for the Ag-PhC and PhC samples. The IQE and LEE of the $\mathrm{Ag}-\mathrm{PhC}$ sample as a function of wavelength are normalized to those of the PhC sample, as also shown in Figure 5. According to the broadband simulation results, the final emitted light spectrum can be obtained through post processing by multiplying the EQE with the actual normalized QW spectrum that is obtained without any Ag NPs nearby [11]. The simulated PL spectra show that the intensity of $\mathrm{Ag}-\mathrm{PhC}$ sample decreases by 2.5 times, which agrees with the experimental result. However, the enhancement is not completely consistent with that of the experimental result, which is attributed to the approximations, including the dipole-QW approximation [9], the single Ag NP approximation [8], and the simplification of a single dipole for the QWs [11]. Moreover, the transmitted diffraction across the Ag NPs may also affect the consistence. The Purcell factor at $545 \mathrm{~nm}$ is calculated as 18.7, which indicates that LSP-QW coupling is strong and that the SER is 
greatly enhanced. However, due to the dissipation by Ag NP [11], the IQE of the Ag-PhC sample is only $54 \%$ of that of PhC sample at $545 \mathrm{~nm}$. Furthermore, the LEE of Ag-PhC is also decreased by a factor of 1.8, as shown in Figure 5B. Obviously, the decreases of both IQE and LEE lead to the suppression of PL intensity.
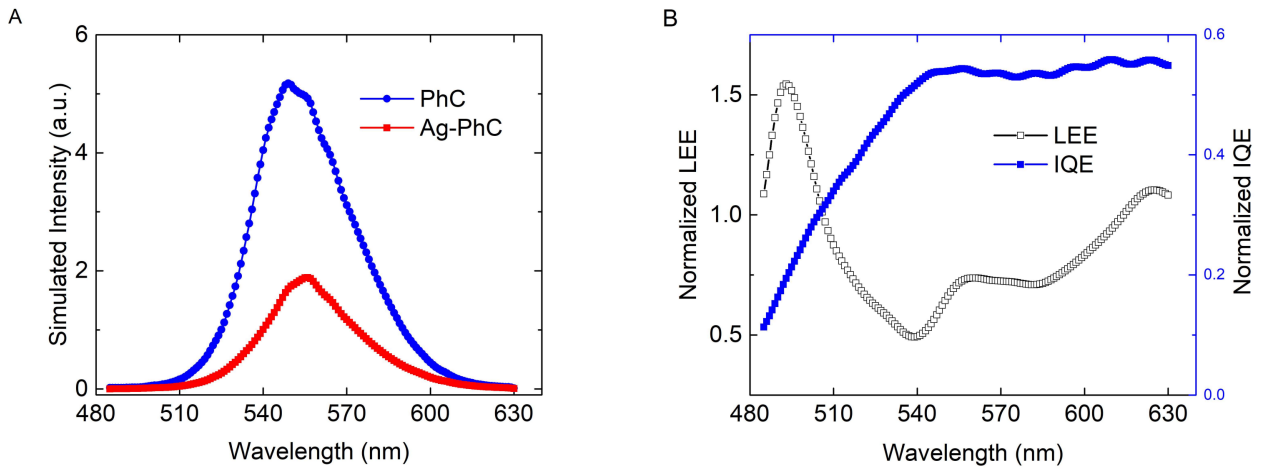

Figure 5. (A) Simulated PL spectra for Ag-PhC and PhC samples, (B) internal quantum efficiency (IQE) and light extraction efficiency (LEE) of Ag-PhC sample normalized to those of the PhC sample.

To simulate the $\mathrm{CL}$ measurement for the $\mathrm{Ag}-\mathrm{PhC}$ sample, the z-dipole is added to the system as shown in Figure 4. Since the e-beam excitation is dependent on its impinging position as discussed above, the simulations are carried out at e-beam impinging points $\mathrm{A}, \mathrm{B}, \mathrm{C}$, and D individually. Considering that the electron beam itself does not radiate light, after a z-dipole is added to simulate the electron beam impinging at specific impinging points, the energy radiated by the z-dipole must be subtracted. With the presence of both the z-dipole and $\mathrm{x}$-dipole, the net powers flowing through the green box, purple box, black box, and the upper red plane monitor $\left(P_{g B o x}, P_{p B o x}, P_{b B o x}\right.$ and $P_{r \text { Plane }}$ respectively) have the relationship of

$$
\begin{gathered}
P_{\text {rPlane }}=P_{\text {up }-x \text { Dipole }}+P_{\text {up }-z \text { Dipole }} \\
P_{p \text { Box }}=P_{x \text { Dipole }} \\
P_{g B o x}=P_{z \text { Dipole }}-\left(P_{A g-x \text { Dipole }}+P_{A g-z \text { Dipole }}\right) \\
P_{b \text { Box }}=P_{x \text { Dipole }}+P_{z \text { Dipole }}-\left(P_{A g-x \text { Dipole }}+P_{A g-z \text { Dipole }}\right)
\end{gathered}
$$

where $P_{x \text { Dipole }}$ and $P_{z \text { Dipole }}$ are the power radiated by the x-dipole and z-dipole, which can be directly recorded in the simulations. $P_{u p}$ is the power extracted upward into the air, and $P_{A g}$ is the dissipated power by Ag NP. These two quantities can also be recorded directly; however, they are the sum of the $x$-dipole and z-dipole components. To distinguish the efficiencies of the $x$-dipole $(\mathrm{QW})$ from the two orthogonal dipoles system, simulations at each point without $x$-dipole have also been performed. Similarly, Equations (3)-(6) without x-dipole component is rewritten as

$$
\begin{gathered}
P_{r \text { Plane }}^{\prime}=P_{u p-x \text { Dipole }}^{\prime}+P_{u p-z \text { Dipole }}^{\prime} \\
P_{p \text { Box }}^{\prime}=P_{x \text { Dipole }}^{\prime}=0 \\
P_{g \text { Box }}^{\prime}=P_{z \text { Dipole }}^{\prime}-P_{A g-z \text { Dipole }}^{\prime} \\
P_{b \text { Box }}^{\prime}=P_{x \text { Dipole }}^{\prime}+P_{z \text { Dipole }}^{\prime}-P_{A g-z \text { Dipole }}^{\prime}
\end{gathered}
$$

where the prime (') indicates all the powers recorded in the case without the $\mathrm{x}$-dipole.

As mentioned above, for the laser beam with a power of $150 \mathrm{~mW}$ and a spot diameter of $\sim 1 \mathrm{~mm}$, the power density of the laser beam is on the order of $\sim 100 \mathrm{~mW} / \mathrm{mm}^{2}$. Additionally, for the e-beam 
with a beam current of $158 \mathrm{pA}$ at $15 \mathrm{kV}$ impinging on the $160 \mathrm{~nm}$ diameter Ag NP, the power density of the e-beam is on the order of $\sim 100 \mathrm{~W} / \mathrm{mm}^{2}$. Considering the dipole-QW and dipole-e-beam approximations and that the energy of e-beam cannot be fully converted to the QW radiation, the ratio of the amplitude of $z$-dipole to that of $x$-dipole was roughly set to be only 10 since the power of a dipole is proportional to the square of its amplitude. By default, the power recorded by different detectors in Equations (3)-(6) and (7)-(10) are normalized to the sum of power from all sources $\left(P_{\text {source }}\right)$. For consistency, all power in Equations (3)-(10) were renormalized by multiplying a correction factor of $P_{\text {source }} / P_{0}$, where $P_{0}$ is defined in Equation (1). Figure 6A clearly shows that $P_{z \text { Dipole }}$ and $P_{z \text { Dipole }}^{\prime}$ vs. wavelength almost coincide in the wavelength range from $480 \mathrm{~nm}$ to $630 \mathrm{~nm}$ at point $\mathrm{B}$. We set $P_{z \text { Dipole }}=(1+\beta) P_{z \text { Dipole, }}^{\prime}$ where $\beta$ is a modification coefficient for the $\mathrm{x}$-diploe. According to the simulation results, $\beta$ is a small quantity. On the contrary, the power of $x$-dipole $\left(P_{x \text { Dipole }}\right)$ changes greatly with the presence of the z-dipole, as shown in Figure 6B. Figure 6C shows the Purcell factor for the $x$-dipole and z-dipole without the Ag NP. It is noted that the direct interaction between x-dipole and $\mathrm{z}$-dipole is far weaker than that shown in Figure 6B. Therefore, the great change in Figure 6B is attributed to the SP strongly excited by z-dipole rather than z-dipole itself. Besides, the peak on the left of $545 \mathrm{~nm}$ in Figure 6B is enhanced with the presence of the $\mathrm{z}$-dipole, indicating that high-order modes in LSP are excited and radiated [13]. According to the simulation, the electric field mapping under the Ag NP also exhibits a quadrupole characteristic at shorter wavelength peak, which again confirms the excitation of the high-order modes.

Table 1 lists the powers of z-dipole and x-dipole at different positions at $545 \mathrm{~nm}$ for Ag-PhC sample. The simulation results at all the points show that the presence of $x$-dipole can hardly affect $z$-dipole whereas the $z$-dipole strongly influences on the $x$-dipole through the excitation of the LSP. Thus, it is reasonable to assume that the dissipated power and extracted power of z-dipole linearly changes with $P_{z \text { Dipole, }}$ that is

$$
\begin{aligned}
& P_{\text {Ag-zDipole }}=(1+\beta) P_{A g-z \text { Dipole }}^{\prime} \\
& P_{\text {up-zDipole }}=(1+\beta) P_{\text {up-zDipole }}^{\prime}
\end{aligned}
$$

By subtracting Equations (4)-(10) from Equations (3)-(6), the effects of x-dipole can be separated from the e-beam-LSP-QW coupling system as follows

$$
\begin{gathered}
P_{x \text { Dipole }}=P_{p B o x} \\
P_{\text {Ag-xDipole }}=P_{g B o x}-(1+\beta) P_{g B o x}^{\prime} \\
P_{\text {up }-x \text { Dipole }}=P_{\text {rPlane }}-(1+\beta) P_{\text {rPlane }}^{\prime}
\end{gathered}
$$
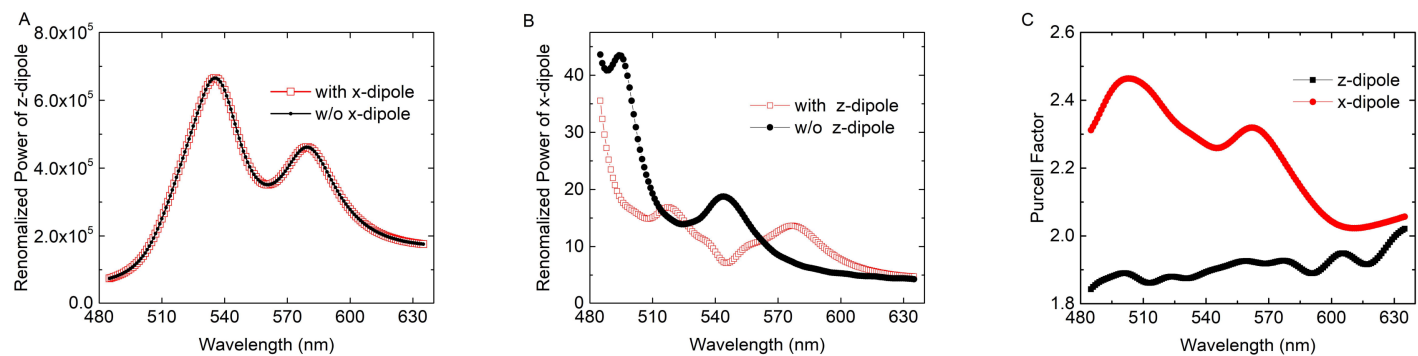

Figure 6. Renormalized powers vs. wavelengths of (A) z-dipole with and without $x$-dipole and (B) $\mathrm{x}$-dipole (i.e., $\mathrm{Fp}$ in this case) with and without $\mathrm{z}$-dipole at impinging point $\mathrm{B}$; (C) Purcell factor for the x-dipole and z-dipole without the Ag NP. 
Table 1. Powers of $\mathrm{z}$-dipole and $\mathrm{x}$-dipole and efficiencies of $\mathrm{x}$-dipole at different positions at $545 \mathrm{~nm}$ for Ag-PhC sample.

\begin{tabular}{cccccccc}
\hline \multirow{2}{*}{ Position } & \multicolumn{2}{c}{ Power of z-Dipole } & \multicolumn{2}{c}{ Power $(F p)$ of x-Dipole } & \multicolumn{2}{c}{ Efficiency of x-Dipole } \\
\cline { 2 - 8 } & $\begin{array}{c}\text { With } \\
\text { x-Dipole }\end{array}$ & $\begin{array}{c}\text { w/o } \\
\text { x-Dipole }\end{array}$ & $\begin{array}{c}\text { With } \\
\text { z-Dipole }\end{array}$ & $\begin{array}{c}\text { w/o } \\
\text { z-Dipole }\end{array}$ & LEE & IQE & EQE \\
\hline A & 189.04 & 196.55 & 11.38 & 18.7 & 2.69 & 1.15 & 3.02 \\
B & 530115 & 530081 & 7.11 & 18.7 & 2.41 & 1.43 & 3.36 \\
C & 1346570 & 1346580 & 15.32 & 18.7 & 1.86 & 1.52 & 2.75 \\
D & 44715.1 & 44427.7 & 17.12 & 18.7 & 0.64 & 0.43 & 0.27 \\
\hline
\end{tabular}

Based on Equations (1), (2), and (13)-(15), the EQE for the $x$-dipole $(\mathrm{QW})$ in the e-beam-LSP-QW system can be obtained. The typical impinging positions $\mathrm{A}, \mathrm{B}, \mathrm{C}$ and $\mathrm{D}$ in the $\mathrm{Ag}-\mathrm{PhC}$ sample are calculated and their weights are considered. The efficiencies of the x-dipole at $545 \mathrm{~nm}$ are also listed in the Table 1. Figure 7A shows the averaged CL spectra of Ag-PhC sample. The CL intensity of the $\mathrm{Ag}-\mathrm{PhC}$ is enhanced by 2.4 times compared with that of the $\mathrm{PhC}$ sample, which agrees with the experimental result. The incomplete consistency is attributed to the approximations, as mentioned above. It is found that the EQE of the x-dipole in most areas around the Ag NP are enhanced by more than 2 times, except at its central area. The EQE at position D is only 0.27 times that of the PhC sample, which agrees with the panchromatic CL images in Figure 3C. In order to better understand the CL enhancement, the EQE at point B was divided to IQE and LEE, as shown in Figure 7B. Both the LEE and the IQE of the $x$-dipole are enhanced in the emission range compared with those of the PhC sample, which is quite different from that of the PL case. The LEE and IQE is enhanced by 2.41 and 1.43 times at $545 \mathrm{~nm}$, respectively. It is notable that the $F_{p}$ of $\mathrm{x}$-dipole at point $\mathrm{B}$ is the smallest in the four points in the CL simulation, as shown in Table 1 . When the $F_{p}$ is enhanced to a certain value, it is no longer important for IQE enhancement. Since the term $\frac{F_{p} \gamma_{r a d}}{F_{p} \gamma_{r a d}+\gamma_{n o n}}$ in Equation (2) approaches 1, IQE is dominated by the scattering ratio (i.e., $\frac{\gamma_{\text {scat }}}{\gamma_{\text {diss }}+\gamma_{\text {scat }}}$ ). Compared with the simulated PL results, the scattering ratio of the x-dipole is enhanced 2.65 times. That is, when the z-dipole is added, less dissipation in the Ag NP can be obtained even when the $F_{p}$ decreases. According to References $[8,13]$, with the presence of the z-dipole, the blue-shifted resonant peak in Figure $6 \mathrm{~B}$ and the enhanced IQE in Figure $7 \mathrm{~B}$ indicates that the original higher-order nonradiating modes and lower-order radiating modes are suppressed and enhanced, respectively. A high $F_{p}$ may lead to severe luminescence quenching effect via the high-order LSP modes [8].
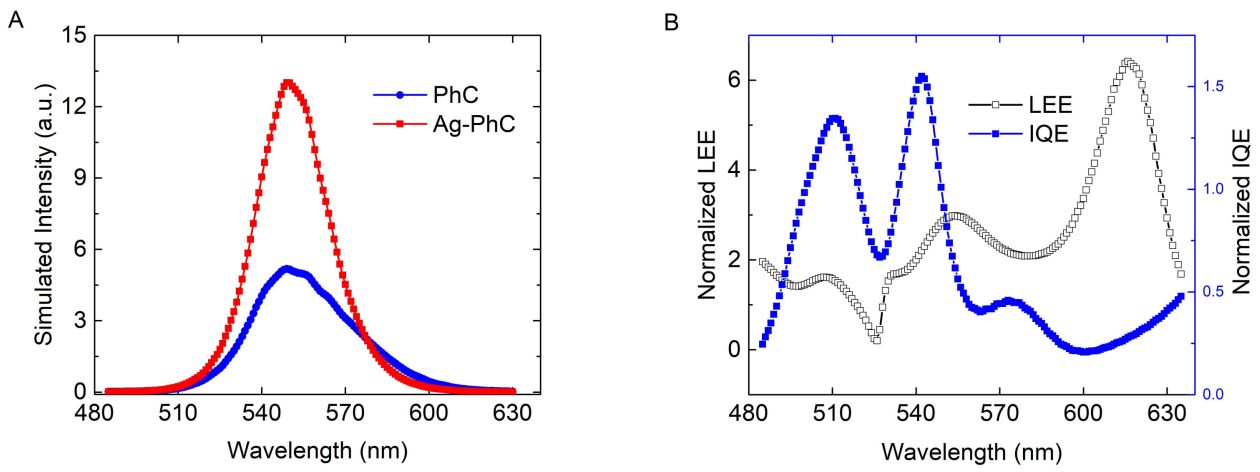

Figure 7. (A) Simulated CL spectra for Ag-PhC and PhC samples; (B) IQE and LEE of x-dipole normalized to those of the $\mathrm{PhC}$ sample at impinging point $\mathrm{B}$ for the $\mathrm{Ag}-\mathrm{PhC}$ sample.

In addition, the LEE in CL simulation is also surprisingly enhanced by 4.3 times at $545 \mathrm{~nm}$ at point B compared with the PL results in Figure 5B. The LEE enhancement of $68 \%$ of the Ag-PhC sample compared with the $\mathrm{PhC}$ sample has been obtained in our previous work, which was explained by the resultant modification of the waveguide mode, which combines the effects of the LSP and the PhC [11]. 
In this work, the enhancement may occur because the excessive LSP modes excited by the z-dipole also modify the waveguide mode in the GaN LEDs. The penetration ability of high-energy electrons is stronger than that of the laser, which will enhance the CL intensity as well.

As described above, the z-dipole introduction enhances both the IQE and LEE of the QWs in the LSP-QWs coupling process. The dissipated energy is reduced and the waveguide modes are extracted effectively. It is reasonable to make an orthogonal emission dipole in LSP-QW coupling systems to enhance the light output of the devices. The InGaN QWs epitaxy on pyramids or V-pit structures seems to provide the possibility of two orthogonal dipoles coupling to LSP [25], where dipole radiators within the quantum wells on the facets are similar with the z-dipole in this work compared with the dipole radiators within normal quantum wells.

\section{Conclusions}

In summary, PL and CL measurements were performed on a green LED with Ag NPs filled in photonic crystal holes. The suppression of PL and the enhancement of CL were observed compared with the PhC sample. In the FDTD simulations, the two orthogonal dipoles were used to couple with the LSP. The x-dipole (QW) effect was separated in the e-beam-LSP-QW system by linear approximation. The simulation showed that both the IQE and the LEE were enhanced by the $\mathrm{z}$-dipole added to the LSP-QW system. The enhancements were attributed to the LSP excited by z-dipole coupled to some LSP modes excited by the x-dipole. A novel LED device was proposed with orthogonal emission dipoles based on the simulation and experimental results.

Acknowledgments: This work was supported by National Key Research and Development Program under Grant No. 2016YFB0400100, National Natural Science Foundation of China under Grant No. 61674005, National Natural Science Foundation of China under Grant No. 61334009, Beijing Municipal Science \& Technology Commission under Grant No. Z161100001616010 and Science and Technology Major Project of Guangdong Province under Grant No. 2016B010111001.

Author Contributions: Y.F. designed the experiment, prepared the samples, performed the measurements and the simulation and wrote the manuscript. Z.C. designed the experiment and wrote the manuscript. S.J. contributed to the conception of the study and the CL measurement. C.L. contributed to the PL measurement. C.L. contributed to the PL measurement. Y.C. contributed to preparing the sample. J.Z. contributed to the PL measurement and data analysis. Y.C. contributed to data analysis. J.N. contributed to preparing the sample. F.J. contributed to preparing the sample and the PL measurement. X.K. contributed to the data analysis. S.L. contributed to preparing the samples. T.Y. contributed to the data analysis. B.S. and G.Z. supervised the study and reviewed the manuscript.

Conflicts of Interest: The authors declare no conflict of interest.

\section{References}

1. Krames, M.R.; Shchekin, O.B.; Mueller-Mach, R.; Mueller, G.O.; Zhou, L.; Harbers, G.; Craford, M.G. Status and future of high-power light-emitting diodes for solid-state lighting. J. Disp. Technol. 2007, 3, 160-175. [CrossRef]

2. Saito, S.; Hashimoto, R.; Hwang, J.; Nunoue, S. InGaN light-emitting diodes on c-face sapphire substrates in green gap spectral range. Appl. Phys. Express 2013, 6, 111004. [CrossRef]

3. Yamamoto, S.; Zhao, Y.; Pan, C.C.; Chung, R.B.; Fujito, K.; Sonoda, J.; DenBaars, S.P.; Nakamura, S. High-efficiency single-quantum-well green and yellow-green light-emitting diodes on semipolar (20-21) GaN substrates. Appl. Phys. Express 2010, 3, 122102. [CrossRef]

4. Hwang, J.; Hashimoto, R.; Saito, S.; Nunoue, S. Effects of local structure on optical properties in green-yellow InGaN/GaN quantum wells. Proc. SPIE 2013, 8625, 455-461.

5. Zhang, J.L.; Xiong, C.B.; Liu, J.L.; Quan, Z.J.; Wang, L.; Jiang, F.Y. High brightness InGaN-based yellow light-emitting diodeswith strain modulation layers grown on Si substrate. Appl. Phys. A 2014, 114, 1049-1053. [CrossRef]

6. Okamoto, K.; Kawakami, Y. High-efficiency InGaN/GaN light emitters based on nanophotonics and plasmonics. IEEE J. Sel. Top. Quantum Electron. 2009, 15, 1199-1209. [CrossRef] 
7. Huang, C.W.; Tseng, H.Y.; Chen, C.Y.; Liao, C.H.; Hsieh, C.; Chen, K.Y.; Lin, H.Y.; Chen, H.S.; Jung, Y.L.; Kiang, Y.W.; et al. Fabrication of surface metal nanoparticlesand their induced surface plasmon coupling with subsurface InGaN/GaNquantum wells. Nanotechnology 2011, 22, 475201. [CrossRef] [PubMed]

8. Sun, G.; Khurgin, J.B. Plasmon enhancement of luminescence by metal nanoparticles. IEEE J. Sel. Top. Quantum Electron. 2011, 17, 110-118. [CrossRef]

9. Gontijo, I.; Boroditsky, M.; Yablonovitch, E.; Keller, S.; Mishra, U.K.; DenBaars, S.P.; Krames, M. Coupling of InGaN quantum-well photoluminescence to silver surface plasmons. Phys. Rev. B 1999, 60, 11564-11567. [CrossRef]

10. Akselrod, G.M.; Argyropoulos, C.; Hoang, T.B.; Ciracì, C.; Fang, C.; Huang, J.N.; Smith, D.R.; Mikkelsen, M.H. Probing the mechanisms of large Purcell enhancement in plasmonic nanoantennas. Nat. Photonics 2014, 8 , 835-840. [CrossRef]

11. Jiang, S.; Chen, Z.Z.; Feng, Y.L.; Jiao, Q.Q.; Fu, X.X.; Ma, J.; Li, J.Z.; Jiang, S.X.; Yu, T.J.; Zhang, G.Y. The coupling behavior of multiple dipoles and localized surface plasmon in Ag nanoparticles array. Plasmonics 2016, 11, 125-130. [CrossRef]

12. Maier, S.A. Plasmonics: Fundamentals and Applications; Springer: New York, NY, USA, 2007.

13. Kuo, Y.; Chang, W.Y.; Chen, H.S.; Kiang, Y.W.; Yang, C.C. Surface plasmons coupling with a radiating dipole near a Ag nanoparticle embedded in GaN. Appl. Phys. Lett. 2013, 102, 161103. [CrossRef]

14. Jiang, S.; Hu, Z.; Chen, Z.Z.; Fu, X.X.; Jiang, X.Z.; Jiao, Q.Q.; Yu, T.J.; Zhang, G.Y. Resonant absorption and scattering suppression of localized surface plasmons in Ag particles on green LED. Opt. Express 2013, 21, 12100-12110. [CrossRef] [PubMed]

15. Chaturvedi, P.; Hsu, K.H.; Kumar, A.; Fung, K.H.; Mabon, J.C.; Fang, N.X. Imaging of plasmonic modes of silver nanoparticles using high-resolution cathodoluminescence spectroscopy. ACS Nano 2009, 3, 2965-2974. [CrossRef] [PubMed]

16. De Abajo, F.J.G. Optical excitations in electron microscopy. Rev. Mod. Phys. 2010, 82, 209-275. [CrossRef]

17. Coenen, T.; Schoen, D.T.; Brenny, B.J.M.; Polman, A.; Brongersma, M.L. Combined electron energy-loss and cathodoluminescence spectroscopy on individual and composite plasmonic nanostructures. Phys. Rev. B 2016, 93, 195429. [CrossRef]

18. Yang, C.; Manjavacas, A.; Large, N.; Nordlander, P. Electron energy-loss spectroscopy calculation in finite-difference time-domain package. ACS Photonics 2015, 2, 369-375.

19. Estrin, Y.; Rich, D.H.; Keller, S.; DenBaars, S.P. Temperature dependence of exciton-surface plasmon polariton coupling in $\mathrm{Ag}, \mathrm{Au}$, and $\mathrm{Al}$ films on $\operatorname{In}_{\mathrm{x}} \mathrm{Ga}_{1-\mathrm{x}} \mathrm{N} / \mathrm{GaN}$ quantum wells studied with time-resolved cathodoluminescence. J. Appl. Phys. 2015, 117, 043105. [CrossRef]

20. Estrin, Y.; Rich, D.H.; Keller, S.; DenBaars, S.P. Observations of exciton-surface plasmon polariton coupling and exciton-phonon coupling in InGaN/GaN quantum wells covered with Au, Ag, and Al films. J. Phys. Condens. Matter 2015, 27, 265802. [CrossRef] [PubMed]

21. Gao, N.; Huang, K.; Li, J.C.; Li, S.P.; Yang, X.; Kang, J.Y. Surface-plasmon-enhanced deep-UV light emitting diodes based on AlGaN multi-quantum wells. Sci. Rep. 2012, 2, 816. [CrossRef] [PubMed]

22. Lumerical Illuminating the Way. 2003. Available online: http://www.lumerical.com (accessed on 8 November 2017).

23. Jiang, S.; Chen, Z.Z.; Fu, X.X.; Jiao, Q.Q.; Feng, Y.L.; Yang, W.; Ma, J.; Li, J.Z.; Jiang, S.X.; Yu, T.J.; et al. Fabrication and effects of Ag nanoparticles hexagonal arrays in green LEDs by nanoimprint. IEEE Photonics Technol. Lett. 2015, 27, 1363-1366. [CrossRef]

24. Sun, G.; Khurgin, J.B. Comparative study of field enhancement between isolated and coupledmetal nanoparticles: An analytical approach. Appl. Phys. Lett. 2010, 97, 263110. [CrossRef]

25. Ajia, I.A.; Edwards, P.R.; Pak, Y.; Belekov, E.; Roldan, M.A.; Wei, N.N.; Liu, Z.Q.; Martin, R.W.; Roqan, I.S. Generated Carrier Dynamics in V-Pit-Enhanced InGaN/GaN Light-Emitting Diode. ACS Photonics 2018, 5, 820-826. [CrossRef]

(C) 2018 by the authors. Licensee MDPI, Basel, Switzerland. This article is an open access article distributed under the terms and conditions of the Creative Commons Attribution (CC BY) license (http:// creativecommons.org/licenses/by/4.0/). 